Schweiz. Z. Path. Bakt. 1946;9:I-IV

\title{
Contents, Vol. 9, 1946
}

Offizielles Organ der Treien Vereinigung Schweizer Pathologen Organe officiel de $\Gamma$ Association libre des Pathologistes Suisses

Offizielles Organ der Schweizerischen Mikrobiologischen Gesellschaft Organe officiel de la Société Suisse de Microbiologie

Unter Mitarbeit von: C. Hallauer P. Hauduroy H. v. Meyenburg J.L.Nicod Th. Reh

Bern Lausanne Zurich Lausanne Geneve

E. Rutishauser J. Tomcsik C. Wegelin A. Werthemann

Geneve Basel Bern Basel

Herausgegeben von:

A. v. Albertini - A. Grumbach - H. Mooser

Zurich

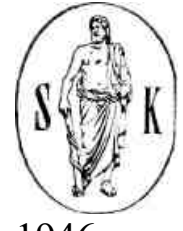

1946

BASEL (Schweiz) S. KARGER NEW YORK

Vol. IX

Alle Rechte vorbehalten

Printed in Switzerland.

Cliches: Aberegg-Steiner \& Cie. AC, Bern.

Druck von Fried rich Reinhardt in Basel.

Index.

Albertini, A. v., Cytologische Exsudatbefuivde mit dem Phasenkonírastverfahren $701 \Lambda \mathrm{mbe} \tau \mathrm{g}$, B., vide Régamey, R.

Arnaudi, C, Flavobaclerium oxydant les stérides à action hormonale . . 607 Baer, J. G., et

Scheidegger, S., Un cas intéressant de iétraplégie d'origine para-

sitaire 61

Berblinger, W., Die tuberkulöse Endophlebitis ... 32

- Zur Pathologic des Hypophyseii-Zwischenhirnsystems $\quad 681$

Berger, E., Konstitution und Spezifität künstlicher Antigene 543

Berger, E., und Brzezinski, St., Die unterschiedliche chemotherapeutische Sen-

sibilität der Gifte eines klassischen und murinen Fleckfieberstammes . 452

Block, H., Adsorplionsphänomene an Tuberkelbazillen 431

Block, H., und: Suter, E., Die Essigsäureatmung von Tuberkelbazillen . . . 434

- $\quad$ Extraction und Nachweis kleiner Peptidasenniengen aus Bakterien . 597

Boivin, A., Travaux récents sur la conslitulioii chimique et sur les propriétés 
biologiques des antigènes baclériens 505

Brzezinski, St.. vide Berger. E.

Bucher, ()., Weitere Untersuchungen über die Wirkung von Colchiein auf Ge-

$\Lambda$ vebekulturen. 4. Der Einlluß von Ascorbínsäure (Vilamin C), Araboascor-

binsäure und $p$-Chinon auf die Colchicinwirkung (543

Buckley. J.J., Über einen außergewöhnlichen Fall von 1·ierdförmigen, enlzünd-

lich bedingten Koagulations-Nekrosen in líirn und Rückenmark als Folge-

erscheinung chronisclur Bronchdekíasen 148

Ettlinger, L.. Antibiose und anlibiotische Stoffe der Pflanzen $\quad 352$

Fischer, R., vide Tomcsik, J.

Flückiger, G., Prophylaxie de la fièvre aphteuse $\quad 592$

Frédéricq, P., Sur la spécificité des actions antibiotiques 385

Freí, W., und Jezierski, A.. Die Wirkung von Elkosin bei der infizierten Maus, auf die Entwicklung und auf Oxydationsprozesse von Bakterien im Glas

im Vergleích mit Cibazol 277

Fust. B., und Grünig, P.. Über den Einlluß des Penicillins auf die Kuhpockenlymphe4-15

Gräub, E., Saxe $\tau$, E., und Vonarburg, IL. Beobachlungen über den Einfluß der

Brucella-Infektion auf die Tu·berkulose beim Rinde 180

Grumbach, A.. Zum Wirkungsmechan'ismus einiger Desinfektionsmillei der Hg-

Reihe... 395

Grünig P.. vide Fust. B.

Guilloud, II.. vide Schopfer, IV. //.

Hallauer, C, Über den Virusnachweis mit dem Hirst-Test 553

Herlant. M., vide Mutsaars, TV.

Heß, W., Zur histologischen Diabelesdiagnose an den Langerhansschen Inseln.

Untersuchungen mit Versilberung nach Gros-Schultze 46

Hoffmann, S., Desinfektionsergebnisse in künstlich erstellten olfenen Baele-

anlagen $\quad 457$

- Zur Baktcriologie hauseingemachter Konser $\Lambda$ en $\quad 582$

Ivert, A., Ein Fall von reiner Myelosklerose 1

Jenny. J., Zur vergleichenden pathologischen Analomie des primären Lungen-

kaГzinoms unler Berücksichtigung eines Falles beim Pí'erd und Hund . 618 Jezierski, A., vide

Freí, W. $1<$ ästli, P., Die Behan·dlung der Slreptokokken-Maslitis (Gelber Gait) der Kühe

mit Penicillin 440

Kradolfer, F.. Die Bedeutung des «spreading-factor» fur das Bild der Pneumo-

kokkenpneumonie

Lüthy, F, und Zollinger, II. U., Beitrag zur Frage der Myelomalacie (Periskle-

rose der Rückenmarksgefäße) $)^{\wedge} 04$

Machebæuf, M.. Travaux récents sur la constitution chimique des anticorps 466 Markees, S,

Über Wachstumsversuche mit Staphylococcus aureus im vollsyn-

thetischen Nährmedium und zur Frage des Pantothensäure-Salicylat-Ant-

agonismus \&f

Meier, R, Zur pharmakologischen Beurteilung der antibiotischen Wirkung . . 323

Meyenburg, II. v., Uraemie-Tod nach elektrischem Unfall 662

Mosímann, W., Bakleriophagen als Ursache von Fabrikationsstörungen in 
Milchverarbeitungsbetriel $>$ en

Mutsaars, W., und Herlant. M.. La bronchopneumonie vacciuale chez le lapin 291 Neipp, L., Contribution a Tétude de l'influence du chlorure de sodium sur

Taction désinfeelante 66

Neukomm, S., Fer, système réticulo-endothélial et échanges dermo-épidermiques (Tis·sus normaux et cancéreux). Avec planches I et II . . . 161

Neumann, J., Su $\gamma$ Гinhibition de croissanoe de $\Gamma$ Aspergillus niger provoquée par le pyrocatéchol et par la p-quinone, similitude de Гantagonisme exercé par Гacide para-amino-benzoïque et par la para-amino-phényl-sulfamide

sur cette inhibition 419

- Sur Гinhibition de croissance de ГAspergillus niger provoquée par la paminophénylsulfamide; suppression de cette inhibition par le phenol et par certains diphénols $\quad$... 426 Nicod, J. L., Hyphomycose (Aspergillose) méningée Pestalozzi, P., Über das Vorkommen und die klinische Bedeutung der Kälteagglutination bei der Viruspneumonie 214

Régamey, R., L'application du phénomène de Seemüller au titrage de Гanatoxine diphtérique 565

Régamey, R., und Amberg, B., Beitrag zur Tetanustoxin-Antitoxin-Flockung nach Ramon 568

Riniker, P., Über den histologischen Bau der congenitalen Oesophagusstenosen 715

Roulet, Ft., Über die Leberzirrhose der schwarzen Bevölkerung Af' rikas . . 666

Rutishauser, E., Oedematöse Polymyositis 709

Saxer, E., vide Gräub, E.

Scheidegger, S., Libman-Sacks Syndrom 669

Scheidegger, S, vide Daer, J. G.

Schopfer, W. H., und Guilloud, H., Sulfamido-résistance chez ГEremothecirm Ashbyii 416

Seemüller, H., Die Fehlbindung und die Zweckbindung des Diphtheric-Anti gens als experimenteller Beitrag zur Lösung der Frage: Wie entsteht das Diphtherie-Antitoxin? 555

Simons, H., Neue Fortschritte in der parasitologischen Feindiagnostik von Blutprotisten (Trypanosomen, Spirochäten, Leptospiren, Plasmodien) . . . 461

Skorpil, F., Zur Geschichte, Klinik und pathologischen Anatomie des eosinophilen Granuloms 233

Stauby H., Häufung schwerster Hepatitisfälle in Basel

Steinmann, J., Le Bactériophage. Sa nature et son emploi thérapeutique Supplementum

Storck, H., Experimentelle Untersuchungen über die Cibazolresistenz von

Gonokokkenstämmen (Dritte Mitteilung: Die Wirkung von Harnstoff und

Marfanil auf Gonokokken $\gamma \eta$

Sluder, A., Zur Frage der Pigmentierung von Nebennierenrindenadenomen und acce|ssorischen Nebennieren 94

Suter, E., vide Block, H.

Tomcsik, J., und Fischer, /?.. Zur chemischen Natur des Forssman-Antigens aus Pferdeherz573

Uehlinger, E., Über Mischstaubpneumokoniosen

692 
Vonarburg, II., vide Gräub, E.

Walthard, B., Zur pathologischen Anatomie der endogenen Pigmentierung . . 711

Waser, P. G.t Ghronisches Mikrotrauma und Praeneoplasie ... . . $] 29$

Wegelin, C, Eigenartige Metastasen beim Lymphosarkom der Schildrüse . . (;87

Welsch, M., Productions d'actinomycétine et d'actinomycine dans les cultures

submergées de divers streptomyces 379

Werthemann, A., Über Beziehungen sirenoider Fehlbildungen zu Schizosomen 707

Wunderly, Ch., Die Senkungsgeschwindigkeit der Erythrocyten als Meßvorgang

íür die I5elvertung der Molekülgröße von Nukleinsäuren 257

Zoilinger, H. V., vide Lüthyi F.

GESELL·SCHAFTSBEBICHTE - SOCIETY TRANSACTIONS - SOCIÉTÉS

Schweizerische Mikrobiologische Gesellschaft. 5. Jahresversammlung in Basel, 22./23. Juni 1946. - Société Suisse de Microbiologie. 5e assemblée annuelle à Bale, les 22 et 23 juin 1946 $-321$

Freie Vereinigung der Schweizer Pathologen. 12. Jahresversammlung in Bern, 29./30. Juni 1946. - Association libre des Pathologistes Suisses. 12e assem blée annuelle a Berne, les 29 et 30 juin 1946661

NECROLOGIA

Prof. Dr. Benedetto Morpurgo $\dagger \quad$ j25

BUCHBESPRECHUNGEN - BOOKS REVIEW - L·IVRES NOUVEAUX . . 255319657 